\title{
Kooperation der Eltern und Kindesschutz
}

\section{Kritische Anmerkungen zu einer Stolperfalle in der Praxis}

Maria Lüttringhaus

Dr. Maria Lüttringhaus ist Diplom-

Sozialpädagogin und Diplom-

Pädagogin. Sie leitet die Beratungs- und

Fortbildungseinrichtung »LüttringHaus:

Institut für Sozialraumorientierung « in

Essen und sie ist freie Mitarbeiterin beim Institut für Stadtteilentwicklung -

Sozialraumorientierte Arbeit und

Beratung (ISSAB) der Universität

Duisburg-Essen.

Internet http://www.luettringhaus.info
Bei Fällen der Gefährdung des Kindeswohls in Familien hat sich die Einteilung in die drei Stufen bewährt, die unterschiedliche Handlungskonsequenzen erfordern. Eine Kooperationsbereitschaft der Personensorgeberechtigten stellt dabei allein kein geeignetes Merkmal zur richtigen Einstufung des konkreten Falles dar.

»Die Eltern haben einen Antrag auf Hilfe zur Erziehung gestellt. Jetzt sind wir im 'grünen«Bereich, also im Freiwilligenbereich. «

"Solange die Familie eine Sozialpädagogische Familienhilfe annimmt, sind wir ja raus aus dem Kinderschutzbereich. "

»Das Kind ist ja im Heim - dann ist es ja nicht mehr im Gefährdungsbereich! «

In meiner Praxis als Trainerin für Case Management und Sozialraumorientierung in der Jugendhilfe werde ich vielerorts mit derartigen Aussagen konfrontiert. Im folgenden Beitrag werde ich deutlich machen, dass es sich dabei um Fehleinschätzungen mit weitreichenden Konsequenzen handeln kann und welche Schlussfolgerungen für das fachliche Handeln gezogen werden können.

\section{Grundlagen der Falleinordnung in der Jugendhilfe}

In den Kommunen, die nach dem Fachkonzept Sozialraumorientierung arbeiten (siehe dazu Hinte, Treeß 2007), haben sich in der Regel drei Arbeitsbereiche etabliert (z. B. in den Großstädten Münster, Saarbrücken, Augsburg, Berlin, Köln, Bonn und in den Landkreisen St. Wendel, Rosenheim, Rendsburg-Eckernförde): Der sogenannte Freiwilligenbereich (bzw. Leistungsbereich) und der Bereich Kindesschutz mit dem Grau- und dem Gefährdungsbereich, die entsprechend der unterschiedlichen Formen der Verantwortung differenziert betrachtet werden (siehe dazu Münder et al.: Frankfurter Kommentar zum $\mathbb{S} 8$ a SGB VIII; S.184). Entscheidend ist, dass sich je nach Falleinord- nung unterschiedliche Handlungskonsequenzen ergeben und eine falsche Einordnung entsprechende Konsequenzen hat (zum Verfahren der Risikoeinschätzung siehe Lüttringhaus/Streich 2010).

Eine Unterteilung in lediglich zwei Bereiche der Jugendhilfe (z. B. Beratungsbereich und Kindesschutzbereich oder Förderbereich/Gefährdungsbereich) reicht nicht aus (siehe dazu auch Sonderausschuss zur Untersuchung des Falles Lea Sophie vom 10.12.2007; Punkt 3.2, Internet http://www.dbsh.de/untersuchung_Lea-Sophie.pdf). Es bedarf einer differenzierteren Falleinordnung, wie sie im Folgenden vorgestellt wird.

1. Der Freiwilligenbereich (bzw. Leistungsbereich): Hier werden Leute zwar oft von anderen Institutionen geschickt und kommen nicht aus eigenem Antrieb, greifen aber letztlich doch freiwillig auf eine Leistung der Jugendhilfe zurück (z. B. eine Beratung beim Allgemeinen Sozialdienst oder eine Hilfe zur Erziehung). Sie könnten sich jederzeit von der Jugendhilfe verabschieden, ohne dass vonseiten der Professionellen Konsequenzen ergriffen werden können (z. B. der Gang zum Familiengericht; siehe dazu ausführlich Lüttringhaus/Streich 2006). Im Freiwilligenbereich sind die Themen, der Wille und ausgehend davon die Ziele der Klientinnen und Klienten handlungsleitend (siehe Lüttringhaus/Streich 2007).

2. Kindesschutz-Graubereich: Während im Freiwilligenbereich der Jugendhilfe die Themen dominieren, die den Betroffenen, also den Personensorgeberechtigten, Jugendlichen und Kindern wichtig sind, geht es im sogenannten Graubereich in erster Linie um zwei Themen, die den Fachkräften gesetzlich vorgegeben sind: (A) die Überprüfung, ob derzeit eine Kindeswohlgefährdung vorliegt, oder (B) dass eine drohende Kindeswohlgefährdung abgewendet wird.

Im »Klärungsbereich « (A) gilt es zu überprüfen, ob aktuell tatsächlich eine Kindeswohlgefährdung vorliegt oder 
nicht, und ob die von Dritten benannten oder den Professionellen selbst bekannten Sachverhalte einem der Gefährdungsbereiche der Jugendhilfe zugeordnet werden können: gesundheitliche Gefährdung, sexueller Missbrauch, körperliche Gewalt oder häusliche Gewalt (u. a. Erleben von massiver Partnerschaftsgewalt), Aufsichtspflichtverletzung, Autonomiekonflikte (u. a. aus kulturellen Differenzen), seelische Verwahrlosung (u. a. durch massive Vernachlässigung). Da geprüft wird, ob derzeit eine Kindeswohlgefährdung tatsächlich vorliegt, wird dieser Teilaspekt des Graubereichs mancherorts auch Klärungsbereich oder Überprüfungsbereich genannt. Nach einer Überprüfung bei der meldenden Person gehen die Fachkräfte ernsthaften Meldungen in der Regel im direkten Kontakt zu den Personensorgeberechtigten nach. "Auch bei anonymen Hinweisen sind die Familien erste Adressaten eines Gewinnens von Information und nicht außen stehende Dritte wie Nachbarn, die Schule, der Kindergarten etc." (Münder et al, Kommentar zum
\ 8a; S. 169; siehe SGB VIII $\$ 62$ Absatz 2 , Satz 1). Fachkräfte der sozialen Dienste »werben bei den Kindern, Jugendlichen, sowie deren Eltern um eine Mitgestaltung des Hilfeprozesses « (Münder et al 2006; S.169), und klären dann ab, inwieweit die Personensorgeberechtigten hierzu bereit sind. Ist die Kooperationsbereitschaft geklärt, erteilen die Fachkräfte - sowohl die des Allgemeinen Sozialdienstes als auch die der Träger bei den Hilfen zur Erziehung - konkrete Aufträge an die Personensorgeberechtigten, die dem Sachverhalt entsprechend den Sinn haben, die vermutete Kindeswohlgefährdung zu überprüfen (siehe dazu Deutscher Verein 2006, S. 495). Bei Aufträgen, die der Überprüfung dienen, sollen Professionelle die Personensorgeberechtigten offensiv und beharrlich zur aktiven Aufklärung anhalten. Der Graubereich (A) soll also zügig abgeschlossen sein, um von einer »Verfolgung « mit andauernd neuen düsteren Prognosen abzusehen. Die Einführung des $\mathbb{8}$ a SGB VIII sollte dazu beitragen, dass die Mitarbeiterinnen und Mitar- beiter der Sozialen Dienste nach Klärung der Situation zum jetzigen Zeitpunkt in den anderen Einrichtungen und Institutionen, des SGB VIII, in denen sich Kinder aufhalten, verlässliche Partner haben, die die Aspekte des Kinderschutzes - nun auch durch eine entsprechende gesetzliche Verpflichtung - berücksichtigen müssen.

Im Graubereich (B) gilt es die »drohende Gefährdung « abzuwenden, bezogen auf bereits vorhandene (geklärte) konkrete Aspekte einer drohenden Kindeswohlgefährdung. Hier liegen konkrete Sachverhalte vor, die in der Risikoeinschätzung in Abwägung zur Problemeinsicht der Personensorgeberechtigten und deren Ressourcen (siehe dazu Lüttringhaus/Streich 2010) noch nicht so drastisch sind, den Fall als Gefährdungsfall einzustufen, die die Jugendhilfe aber immerhin verpflichten, »am Ball zu bleiben «. Gerichte können dieses "Dranbleiben « bei fehlender Kooperation anordnen: Familien werden dann in verpflichtet, Familienhilfe anzunehmen und mit dem Jugendamt zusammenzuarbeiten. Bei drohender Ge-

\section{Kindesschutz: Ressourcenorientiertes Vorgehen im Graubereich in Kooperation mit den Personensorgeberechtigten}

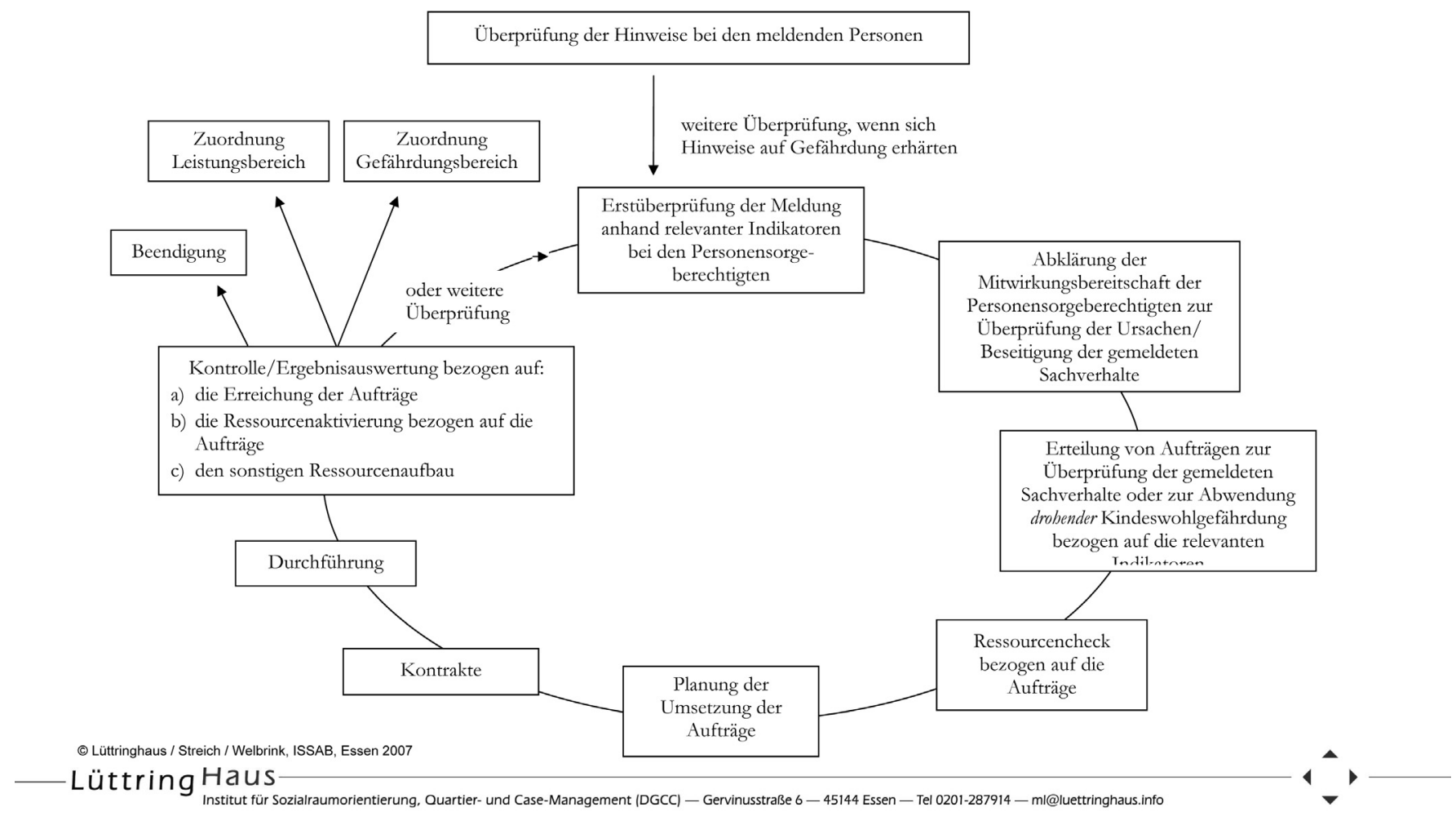

In der Fallarbeit der Jugendhilfe müssen unterschiedliche Formen der Verantwortung beim professionellen Kindesschutz unterschieden werden. Bewährt hat sich die Einteilung in die drei Arbeitsbereiche Freiwilligenbereich, Graubereich und Gefährdungsbereich, die unterschiedliche Handlungskonsequenzen erfordern. Eine Kooperationsbereitschaft der Personensorgeberechtigten ist selbstverständlich immer anzustreben, entlastet aber nicht von einem strukturierten Vorgehen, wie das Beispiel »Graubereich « zeigen kann. 
fährdung haben die Professionellen durch entsprechende Hilfsangebote, darauf hinzuwirken, dass die in den Aufträgen beschriebenen zukünftigen Mindestzustände, die den Kindesschutz sicherstellen, von den Personensorgeberechtigten umgehend angestrebt werden oder weiter sichergestellt werden.

In beiden Fällen ( $\mathrm{A}$ und $\mathrm{B}$ ) werden im Graubereich Aufträge erteilt (zu den Standards von Aufträgen und Auflagen siehe Lüttringhaus/Streich 2008). Kooperieren die Eltern nicht, hat das zunächst » weichere« Konsequenzen als im Gefährdungsbereich. Wenn die Personensorgeberechtigten sich verweigern (z. B. bei der Kontaktaufnahme), dann erfolgt eine Mitteilung an das Gericht als derjenigen Instanz, die dann bei diesen Sachverhalten die entsprechenden Weisungen aussprechen kann (oftmals die oben genannte Anordnung, dass die Familie mit dem Jugendamt kooperieren muss oder unklare Sachverhalte aufzuklären sind, beispielsweise durch Gutachten oder ärztliche Diagnosen).

3. Kindesschutz-Gefährdungsbereich: Hier ist geklärt, dass gegenwärtig konkrete gewichtige Anhaltspunkte für eine Kindeswohlgefährdung in den relevanten Bereichen des Kindesschutzes vorliegen (s. o.). Hier werden von Seiten der Jugendhilfe Auflagen, Anordnungen oder Anweisungen erteilt; »wenn die Eltern nicht gewillt sind oder in der Lage sind, die Gefahr abzuwenden « (Peifer 2008, S. 396) erfolgt der Gang zum Familiengericht, das die Auflagen dann in Form von Geboten, Weisungen, Verboten oder durch Anordnung anderer Maßnahmen erteilt (vgl. Peifer 2008).

\section{Kooperationsbereitschaft und Fremdunterbringung als Stolper- steine bei der Falleinordnung}

Im Arbeitsalltag der Fachkräfte der Sozialen Arbeit zeigen sich die eingangs genannten Stolpersteine bei der Einordnung der Fälle in die Arbeitsbereiche: Eine Fremdunterbringung oder die Kooperation der Personensorgeberechtigten werden oftmals als Indikator gewählt, den Fall als »Freiwilligenfall « zu bewerten:

- »Das Kind ist ja nun in Sicherheit, weil es untergebracht ist. « Was aber gilt am Wochenende, wenn es nach Hause fährt? Was, wenn die Eltern sich entscheiden das Kind zurückzuholen?

- "Solange eine Familienhilfe drin ist, kann ja nichts passieren! «Was aber, wenn die nicht mehr angenommen wird, wenn die Termine unzureichend wahrgenommen werden? Ist den Eltern klar, dass sie selbst verpflichtet sind, den Schutz ihres Kindes zu gewährleisten? Wird klar ausgesprochen, welche konkreten Sachverhalte geändert und in welcher Form sicherzustellen sind?

- »Ja, wenn die Eltern nicht mehr mitziehen, dann wären wir wieder im Graubereich oder Gefährdungsbereich und würden Aufträge oder Auflagen erteilen! « So meist die Antwort der Fachkräfte.

Nun gilt (wie oben beschrieben) grundsätzlich:

a) Ohne die Kooperation der Personensorgeberechtigten gäbe es auch im Grau- und Gefährdungsbereich kein Vorgehen mit den Personensorgeberechtigten (in der Regel erfolgt dann im Gefährdungsbereich bei akuter Ge-

\section{Wie man »Fälle« richtig einordnet}

Die Beantwortung Frage, ob eine bestimmte Fallkonstellation in den »Freiwilligenbereich «, in den »Graubereich « oder in den «Gefährdungsbereich « eingeordnet werden müssen, ist von entscheidender Bedeutung für das konkrete Handeln in der Praxis. Hilfreiche Kontrollfragen für Fachkräfte sind:

- »Würde ich zum jetzigen Zeitpunkt beim Umzug der Familie bei dem dann zuständigen Jugendamt eine Meldung machen (selbst wenn mir die Familie wegen Datenschutz mit dem Rechtsanwalt droht)? «

- »Würde ich beim Aufkündigen der Kooperation den Fall sofort in den Grau- und Gefährdungsbereich einordnen?«

- »Würde ich mich in dem Fall bei einer stationären Unterbringung gegen Rückführungsansprüche der Eltern wehren? Würde ich den Fall dann wieder in den Grau- oder Gefährdungsbereich einordnen und die stationäre Unterbringung weiter als Maßnahme anordnen? «

Falls die Fragen bejaht werden, wären die Fälle im Grau- oder Gefährdungsbereich einzuordnen!

Maria Lüttringhaus

fährdung die Inobhutnahme oder die Anrufung des Familiengerichts und im Graubereich die Klärung ohne die Personensorgeberechtigten bzw. die Mitteilung an das Gericht mit der Aufforderung ein Gutachten, Familienhilfe etc. anzuordnen). Die Kooperationsbereitschaft (incl. Problemeinsicht) und Annahme einer Hilfe sind somit lediglich Indikatoren, ob in dem Familiensystem weitergearbeitet werden kann. Sie beeinflussen darüber hinaus natürlich auch den Grad der Risikoeinschätzung bei der Falleinordnung (siehe dazu Lüttringhaus/Streich 2010).

b) Wenn eine Gefahrenlage beseitigt ist, heißt das nicht, dass »der Fall « raus ist aus dem Grau- oder Gefährdungsbereich. Um es etwas zu überspitzen: Die Jugendhilfe muss immer dafür sorgen, dass eine Gefahrenlage beseitigt wird, sobald sie von einer Gefahrenlage Kenntnis hat und »dran « ist. Das ist eine Kernaufgabe im Bereich Kindesschutz, für die sie bis zur Maßnahme der Inobhutnahme zurückgreifen kann. Würde die Beseitigung der Gefahrenlage ein Indikator sein für die Einordnung in den Leistungsbereich, gäbe es nur sehr kurzfristige Gefährdungsfälle.

Dort, wo Fälle dennoch aufgrund der Kooperation der Eltern in den Freiwilligenbereich eingeordnet werden, müsste die Zielerarbeitung der Eltern im Vordergrund stehen. Es handelt sich aber weiterhin in erster Linie um Themen der Professionellen, die unabhängig der jetzigen Einsicht und Mitarbeit der Eltern am Ball bleiben müssen - auch um zu kontrollieren, inwieweit eine nachhaltige, verlässliche Änderung zu den festgestellten Sachverhalten eintritt.

Dementsprechend müssten diese »Fälle« solange - auch unter Rückgriff auf Kontrollen - im Grau- oder Gefährdungsbereich bleiben, bis der Kinderschutz in der Familie so verlässlich gesichert ist, dass sich die Jugendhilfe zurückziehen kann. Für die Beurteilung des Einzelfalles bieten sich entsprechende Kontrollfragen an (vgl. Kasten »Wie man `Fälle` richtig einordnet $«)$.

Gerade bei Fremdunterbringungen stoße ich häufig auf das Phänomen, dass Fälle vor allem dann dem Freiwilligenbereich zugeordnet werden, wenn das Kind in einer Einrichtung in Sicherheit ist. Ist die Unterbringung jedoch eine verpflichtende 
Maßnahme, um das Kindeswohl zu sichern, ist das Kind zwar in Sicherheit, der Fall bleibt aber solange im Bereich Kindesschutz, solange sich an der gefährdenden Situation im Kontext der Personensorgeberechtigten nichts ändert oder sich noch nichts verlässlich und nachhaltig geändert hat.

\section{Praxisbeispiel: Wie man Auflagen richtig formuliert}

Im Folgenden skizziere ich - reduziert auf das Wesentliche - ein Fallbeispiel, bei dem es bei einer Einordnung in den Gefährdungsbereich blieb - trotz erfolgter Unterbringung und großer Kooperationsbereitschaft. Es soll helfen die eben genannten Ausführungen beispielhaft zu untermauern.

Sachverhalte in den Gefährdungsbereichen: Aufsichtspflichtverletzung, häusli- che Gewalt, Aufsichtspflichtverletzung, gesundheitliche Gefährdung

1. Karin, ein 12-jähriges Mädchen (mit alleinsorgeberechtigter 34-jähriger Mutter) muss die Zeit nach Schulschluss (oft schon ab circa 12.30 Uhr) bis 19.00 Uhr außerhalb der Wohnung verbringen, wobei nichts für sie organisiert wird. Dies wurde von einer Nachbarin gemeldet (für die letzten sechs Wochen mindestens dreimal pro Woche beobachtet); laut Aussage des Mädchens ist »die Mama da immer weg " und in der Regel erst wieder um 19.00 Uhr da. Der Lebensgefährte sagte der Fachkraft des Allgemeinen Sozialdienstes: "Das Mädchen kriegt keinen Schlüssel, damit sie nicht alleine in der Wohnung ist. « Von der Mutter ist bekannt, dass sie suchtkrank ist (alkoholabhängig).

2. Die Personensorgeberechtigte akzeptiert, dass Karin in der Kneipe, in der

\section{Literatur}

Deutscher Verein (Hg.): Empfehlungen des deutschen Vereins zur Umsetzung des \8a SGB VIII. In: Nachrichtendienst des Deutschen Vereins 11/2006, S. 494-501.

Hinte, Wolfgang/Treeß, Helga: Sozialraumorientierung in der Jugendhilfe. Theoretische Grundlagen, Handlungsprinzipien und Praxisbeispiele einer kooperativ-integrativen Pädagogik. Weinheim/München 2007.

Löcherbach, Peter/Mennemann, Hugo/Hermsen, Thomas (Hg.) (2009): Case Management in der Jugendhilfe. Ernst-Rheinhardt Verlag, München/Basel.

Lüttringhaus, Maria/Streich, Angelika (2010): Das Modell der Kurzberatung zur Risikoeinschätzung. In: ISS (Hg.): Der Allgemeine Soziale Dienst. Aufgaben, Zielgruppen Standards. Ernst-Reinhardt, München/Basel. (Im Erscheinen).

Lüttringhaus, Maria/Streich, Angelika (2007): Kindesschutz in der Jugendhilfe. Wie man Auflagen und Aufträge richtig formuliert. Blätter der Wohlfahrtspflege 4/2007, S. $145-150$.

Lüttringhaus, Maria/Streich, Angelika: Zielvereinbarungen in der Sozialen Arbeit: Wo kein Wille ist, ist auch kein Weg. In Gillich, Stefan (Hg.): Nachbarschaften und Stadtteile im Umbruch, Gelnhausen 2007, S. 135-149.

Merchel, Joachim: Garantenstellung und Garantenpflicht: die Schutzfunktion des Jugendamtes zwischen Strafrecht, medialer Öffentlichkeit und fachlichen Konzepten. In: Recht der Jugend und des Bildungswesens (RdJB) 4/2005, S. 456-471.

Merchel, Joachim/Schone, Reinhold: Vereinbarungen mit Trägern von Einrichtungen und Diensten gemäß $\ 8$ a Abs. 2 SGB VIII. In: Forum Erziehungshilfen, 12. Jg., Heft 2, S.109-114.

Münder, Johannes/Baltz, Jochem/Kreft, Dieter/Lakies, Thomas/Meysen, Thomas/Proksch, Roland/Schäfer, Klaus/Schindler, Gila/Struck, Norbert/Tammes, Britta/Trenczeck, Thomas: Frankfurter Kommentar zum SBG VIII: Kinder- und Jugendhilfe. 5. vollständig überarbeitete Auflage 2006. Weinheim/München 2006.

Pfeifer, Ulrike: Die Neuerungen im Bereich des familienrechtlichen Verfahrens. In: Nachrichtendienst des Deutschen Vereins 10/2008, S. 395-400.

Wiesner, Reinhard: Zur Garantenpflicht des Jugendamtes in Fällen der Kindeswohlverletzung. In: Verein für Kommunalwissenschaften e. V. (Hg.) ».... und schuld ist im Ernstfall das Jugendamt«. Aktuelle Beiträge Heft 17, Berlin 1999, S. 7-20.

sie arbeitet, oder auch zu Hause Alkohol trinkt (Bier und Alcopops).

3. Es liegen zwei aktuelle polizeiliche Meldungen über massive häusliche Gewalt vor zwischen ihr und dem Lebensgefährten.

4. In der Wohnung wurden Pornomedien (Hefte, DVDs) gefunden - zugänglich für Karin. Karin zeigt sexualisiertes Verhalten und lud eine Freundin ein, Pornos zu schauen (» Mama kriegt eh oft nix mit.«). Auf Nachfrage zeigt sich, dass sie schon alleine entsprechende DVDs angeschaut hat.

Die Fachkraft formulierte darauf basierend folgende Auflagen, also die zukünftigen Zustände, die es aus Sicht der Fachkräfte bei Karin sicherzustellen gilt.

$\mathrm{Zu}$ 1. "Sie als Mutter müssen sicherstellen, dass Karin ab sofort für die Zeit nach Schulschluss bis 19.00 Uhr einen Ort hat, wo sie vor Wind und Wetter geschützt ist, wo sie was zu Essen und Trinken bekommt, ihre Hausaufgaben machen kann und einen vertrauten Ansprechpartner hat, der verlässlich für sie $\mathrm{da}$ ist."

Zu 2.: »Sie als Mutter sorgen ab sofort dafür, dass Karin in ihrem Beisein oder ihrem Wissen keinen Alkohol mehr trinkt, sondern ausschließlich alkoholfreie Getränke. «

Zu 3.: »Sie als Mutter müssen ab sofort ihre Tochter davor schützen, dass sie die gewalttätigen Ausschreitungen zwischen Ihnen und Ihrem Partner nicht mitbekommt. Im Beisein von Karin müssen Auseinandersetzungen gewaltfrei, beispielsweise mit Worten geregelt werden (nicht mit schlagen). «

$\mathrm{Zu}$ 4.: »Sie als Mutter müssen dafür sorgen, dass ab sofort Pornos durch Sie und ihren Lebensgefährte unzugänglich für Karin aufbewahrt sind."

Terminierung: Alles gilt kontrolliert zunächst für mindestens sechs Monate.

Die Mutter zeigt eine große Problemeinsicht und stellte einen Antrag auf Hilfe zur Erziehung. Ihr spezieller Wunsch war eine Unterbringung der Tochter. Aufgrund der hohen Überforderungssituation und dem Wunsch des Mädchens, woanders zu wohnen, wurde diese Maßnahme auch von der Fachkraft und dem beratenden Team befürwortet. Karin kam in eine Mädchenwohngruppe. Die Mutter plant eine Therapie.

Die Gefahrenlage ist nun beseitigt. Im Rahmen der Arbeit mit Karin erarbeitet 
die Bezugsbetreuerin mit Karin Ziele. Dennoch bleibt der Fall mit Blick auf die Personensorgeberechtigten im Gefährdungsbereich. Die Unterbringung war (und ist noch immer!) eine verbindliche Maßnahme um alle vier Auflagen bezogen auf die vier Sachverhalte der Kindeswohlgefährdung abzusichern. Für die Wochenendkontakte bzw. Kontakte zur Mutter gelten die erteilten Auflagen. Im Rahmen der Elternarbeit gilt es also zu erarbeiten, wie die Auflagen dann dort umgesetzt werden können. Die stationäre Einrichtung hat zudem nach den Kontakten zur Mutter einen Kontrollauftrag.

\section{Folgen der falschen Einordnung in den Freiwilligenbereich}

Werden Fälle wie dieser oder die oben genannten aufgrund der Kooperation, der Beseitigung der aktuellen Gefahrenlage oder einer erfolgten Unterbringung fälschlicherweise in den Leistungsbereich eingeordnet, hat das unterschiedliche Folgen:

1. Fachliche Dimension: Das fachliche Handeln wird für die unterschiedlichen Beteiligten unklar (z. B. unklare oder fehlende Auflagen oder Anweisungen an die
Personensorgeberechtigten, fehlende Kontrollaufträge an die beauftragten Träger, nicht berücksichtigter Zeitaufwand für erhöhte Elternarbeit u. a. auch für Kontrollen und Absprachen). In der Folge bleibt auch die Einhaltung des Kindesschutzes unklar. Zudem werden Rückführungen erschwert, da aufgrund der unklaren Auftragslage an den stationären Träger, die Wissenslage über die aktuelle Veränderung der Situation zu Hause schwerer einzuschätzen ist. Zudem wird der Freiwilligenbereich » verwässert «: Wo Auflagen und Aufträge mit Zielen vermengt werden, leistet das der Tendenz Vorschub, den Willen der Betroffenen und deren Themen unter den Tisch fallenzulassen - auch in » reinen « Leistungsbereichsfällen!

2. Politische Dimension: Eine derartige Praxis, bei der die genannten Fälle in den Freiwilligenbereich eingeordnet werden, führt zu Schieflagen bei der Bewertung der Fallzahlen. Da werden in einer Kommune beispielsweise 90 Prozent der Heimunterbringungen offiziell in den Akten als Freiwilligenfälle geführt (obwohl dies bei weitem nicht so wäre, würde man die oben aufgeführten Grundlagen der Falleinordnung berücksichtigen). Oder es wird im ambulanten Bereich den Leis- tungserbringern vorgerechnet, dass sie ja fast nur Fälle im Freiwilligenbereich haben, aber es doch erstaunlich sei, wieso die Stundenzahl im vergangenen Jahr so angestiegen sei. (Dabei gab es einen rasanten Anstieg von Kindesschutzfällen mit entsprechendem höherem Zeitaufwand!) Eine derartige Praxis verwässert die Entwicklung im Bereich Kindesschutz und dient nicht dazu, die tatsächlichen Dimensionen in der Arbeit im Bereich Kindesschutz widerzuspiegeln. Dies führt mancherorts zu ungerechtfertigten »Deckelungsdiskussionen «, unrealistischen Sparvorgaben und Frust bei den Mitarbeiterinnen und Mitarbeitern, die ihre Arbeit in Belangen des Kindesschutzes nicht ausreichend gewürdigt sehen.

Im Rahmen meiner Fortbildungsreihen insbesondere im Bereich Kindesschutz haben sich mittlerweile viele Kommunen und Einrichtungen dieser Argumentation angeschlossen. Dies war oft verbunden mit zunächst kontroversen internen letztlich fachlich sehr befruchtenden - Diskussionen. Sollten diese Ausführungen auch bei anderen Jugendämtern und Einrichtungen ein Nachdenken auslösen und dann zu einer größeren Klarheit im Bereich Kindesschutz beitragen, hat der Artikel seinen Zweck erfüllt.

\section{Modernisierung sozialer Dienstleistungen}

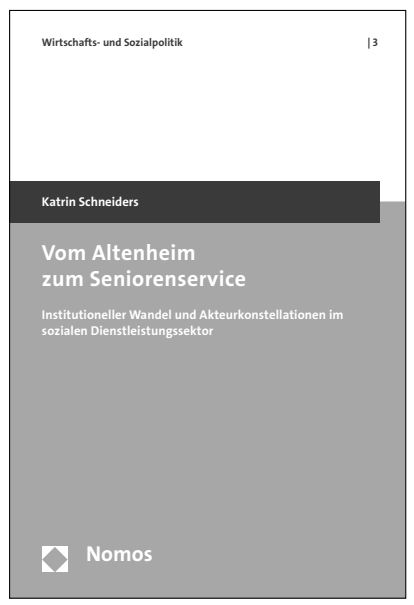

Bitte bestellen Sie im Buchhandel oder

versandkostenfrei unter $\downarrow$ www.nomos-shop.de

\author{
Vom Altenheim zum Seniorenservice \\ Institutioneller Wandel und Akteurkonstellationen im \\ sozialen Dienstleistungssektor \\ Von Dr. Katrin Schneiders
}

2010, 268 S., brosch., 54,- $€$, ISBN 978-3-8329-5347-8

(Wirtschafts- und Sozialpolitik, Bd. 3)

Ökonomisierung, Professionalisierung und Ambulantisierung prägen seit den 199oer Jahren die Altenhilfe, insbesondere den Pflegesektor. Unter Einbezug veränderter Anforderungen der Älteren an Wohn- und Pflegeformen wird diskutiert, welche Rollen etablierte und neue Akteure im zukünftigen Welfare Mix übernehmen können. 